\title{
Heartbeat: The ongoing controversy of intervention for chronic total coronary occlusions
}

doi:10.1136/heartjnl-2018-313921

Catherine M Otto

In patients undergoing primary percutaneous intervention (PCI) for ST-elevation myocardial infarction (STEMI), about $10 \%$ also have a chronic total occlusion (CTO) of a non-culprit coronary artery. It remains controversial whether PCI of the CTO vessel, in addition to the culprit vessel, improves clinical outcome. The results of the randomised Evaluating Xience and left ventricular function in PCI on occlusiOns afteR STEMI (EXPLORE) study of 302 STEMI patients with successful PCI of the culprit vessel are reported in this issue of Heart. ${ }^{1}$ Overall, there was no difference in major adverse cardiac events (MACE) at a median follow-up of 3.9 years in those with or without PCI of the CTO vessel (13.5\% vs $12.3 \%$, HR 1.03 , 95\% CI 0.54 to 1.98 ; $\mathrm{p}=0.93$, figure 1 ). Although all-cause mortality did not differ between groups, cardiac mortality was higher with CTO-PCI (6.0\% vs $1.0 \%, \mathrm{p}=0.02)$. More patients with CTO-PCI were free of angina at 1 year $(6.0 \%$ vs $1.0 \%, p=0.02)$ but his difference was not sustained on longer term follow-up. Overall, CTO-PCI in the setting of STEMI was not associated with a reduction in MACE, improvement in ventricular function or sustained reduction in symptoms.

In the accompanying editorial, Stojkovic and Milasinovic ${ }^{2}$ point out that the rational for CTO-PCI 'rests on the notion of achieving percutaneous complete revascularisation in patients with significant coronary artery disease as incomplete revascularisation has been linked to impaired prognosis.' Although the editorial indicates that 'the authors of the EXPLORE trial are to be congratulated for completing one of the pioneering randomised studies of PCI versus conservative strategy for CTO,' they also express concerns about several limitations of the study design. They go on to recommend that in addition to ongoing randomised trials of CTO, 'potential novel grounds may be explored to accumulate evidence regarding optimal CTO treatment, such

Correspondence to Professor Catherine M Otto, Division of Cardiology, University of Washington, Seattle,WA 98195, USA; cmotto@uw.edu, cmotto@u. washington.edu

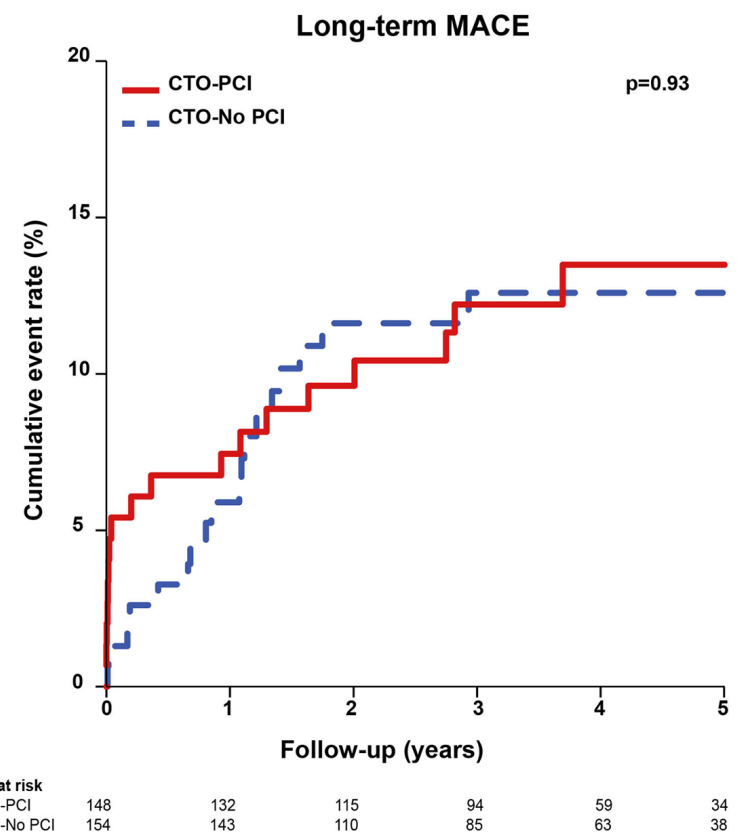

Figure 1 Clinical effect of early CTO-PCI vs CTO-no PCI on MACE in patients with ST-elevation myocardial infarction with a concurrent СTO: long-term follow-up. Kaplan-Meier estimates of the cumulative event rate of the composite end point of MACE: cardiac death, CABG and MI. Analyses were performed on a intention-to-treat basis. CTO, chronic total occlusion; $C A B G$, coronary artery bypass graft; MACE, major adverse cardiac events; MI, myocardial infarction; PCl, percutaneous coronary intervention.

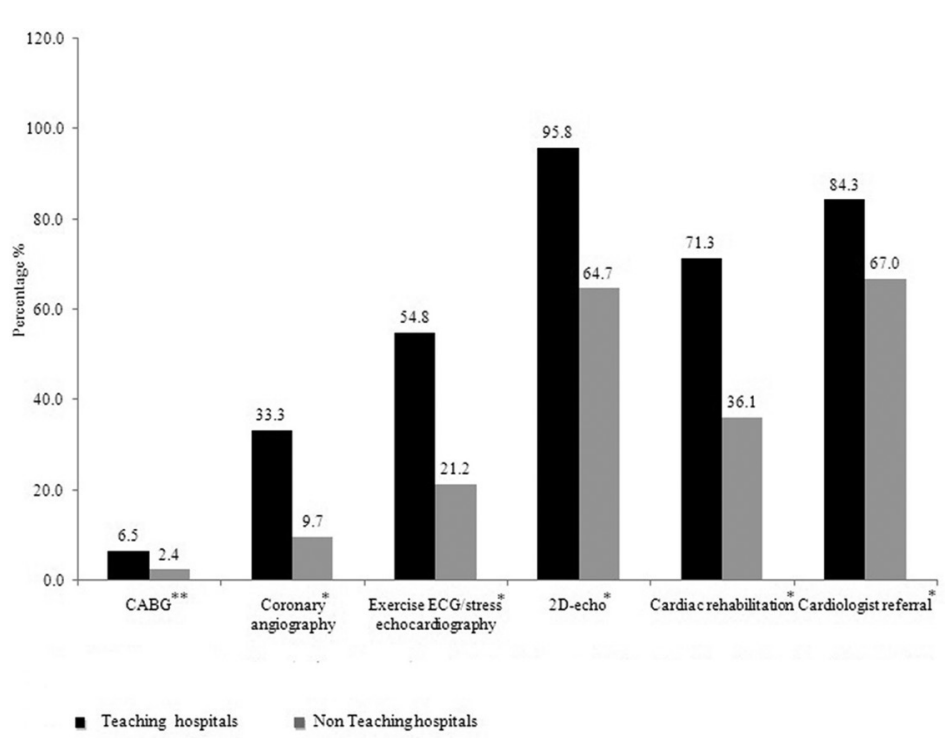

Figure 2 Cardiac interventions/investigations done or planned prior to discharge in teaching versus non-teaching hospitals in acute coronary syndrome ( ${ }^{*} P<0.001$, $\left.{ }^{*} P<0.01\right) .2 \mathrm{D}$, twodimensional; $C A B G$, coronary artery bypass grafting. 


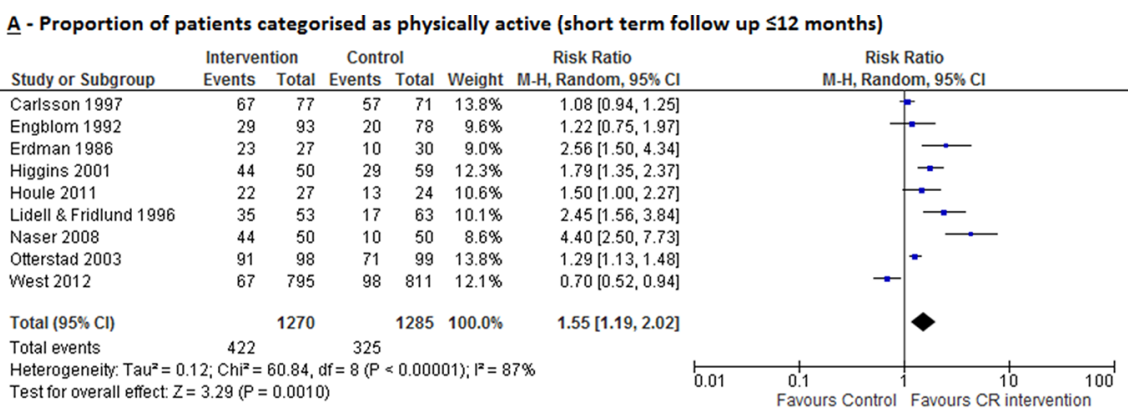

$\underline{B}$ - Proportion of patients categorised as physically active (long term follow up $>12$ months)

\begin{tabular}{|c|c|c|c|c|c|c|c|c|c|c|}
\hline \multirow[b]{2}{*}{ Study or Subgroup } & \multicolumn{2}{|c|}{ Intervention } & \multicolumn{2}{|c|}{ Control } & \multirow[b]{2}{*}{ Weight } & \multirow{2}{*}{$\begin{array}{c}\text { Risk Ratio } \\
\text { M-H, Random, } 95 \% \mathrm{Cl}\end{array}$} & & \multirow{2}{*}{\multicolumn{2}{|c|}{$\begin{array}{c}\text { Risk Ratio } \\
\text { M-H, Random, } 95 \% \mathrm{Cl}\end{array}$}} & \\
\hline & Events & Total & Events & Total & & & & & & \\
\hline Erdman 1986 & 14 & 27 & 10 & 30 & $11.7 \%$ & $1.56[0.83,2.90]$ & & & $\because$ & \\
\hline Lidell \& Fridlund 1996 & 22 & 53 & 17 & 63 & $17.0 \%$ & $1.54[0.92,2.58]$ & & & & \\
\hline Otterstad 2003 & 66 & 98 & 46 & 99 & $71.3 \%$ & $1.45[1.13,1.87]$ & & & 7 & \\
\hline Total $(95 \% \mathrm{Cl})$ & & 178 & & 192 & $100.0 \%$ & $1.48[1.19,1.83]$ & & & $\bullet$ & \\
\hline Total events & 102 & & 73 & & & & & & & \\
\hline $\begin{array}{l}\text { Heterogeneity: Tau }{ }^{2}=0 \\
\text { Test for overall effect: } Z\end{array}$ & $\begin{array}{l}00 ; \mathrm{Ch}^{2}= \\
=3.58(\mathrm{P}=\end{array}$ & $\begin{array}{l}0.07, \mathrm{df} \\
0.0003\end{array}$ & $\begin{array}{l}f=2(P= \\
3)\end{array}$ & $196) ; 1^{2}$ & $=0 \%$ & & 0.0 & $\begin{array}{l}0.1 \\
\text { Favours control }\end{array}$ & 1 Favours CR & $\begin{array}{l}10 \\
10 \\
R \text { intervention }\end{array}$ \\
\hline
\end{tabular}

Figure 3 Impact of cardiac rehabilitation on proportion of patients categorised as physically active measured at (A) short-term follow-up ( $\leq 12$ months) and (B) long-term follow-up (>12 months). CR, cardiac rehabilitation.

as (1) enlarging the network of peers capable of performing high-quality CTO PCI with success rates $>90 \%$ through dedicated proctoring programmes, (2) standardising procedural and outcome reporting to facilitate comparisons among trials and creation of large databases and (3) investigating the effects of CTO PCI in specific disease subsets, such as low EF, where significant improvements have been shown in an observational setting.'

On a worldwide basis, treatment of acute coronary syndromes (ACS) is focused on ensuring that all patients receive known effective therapies including prompt revascularisation and medical therapy. An

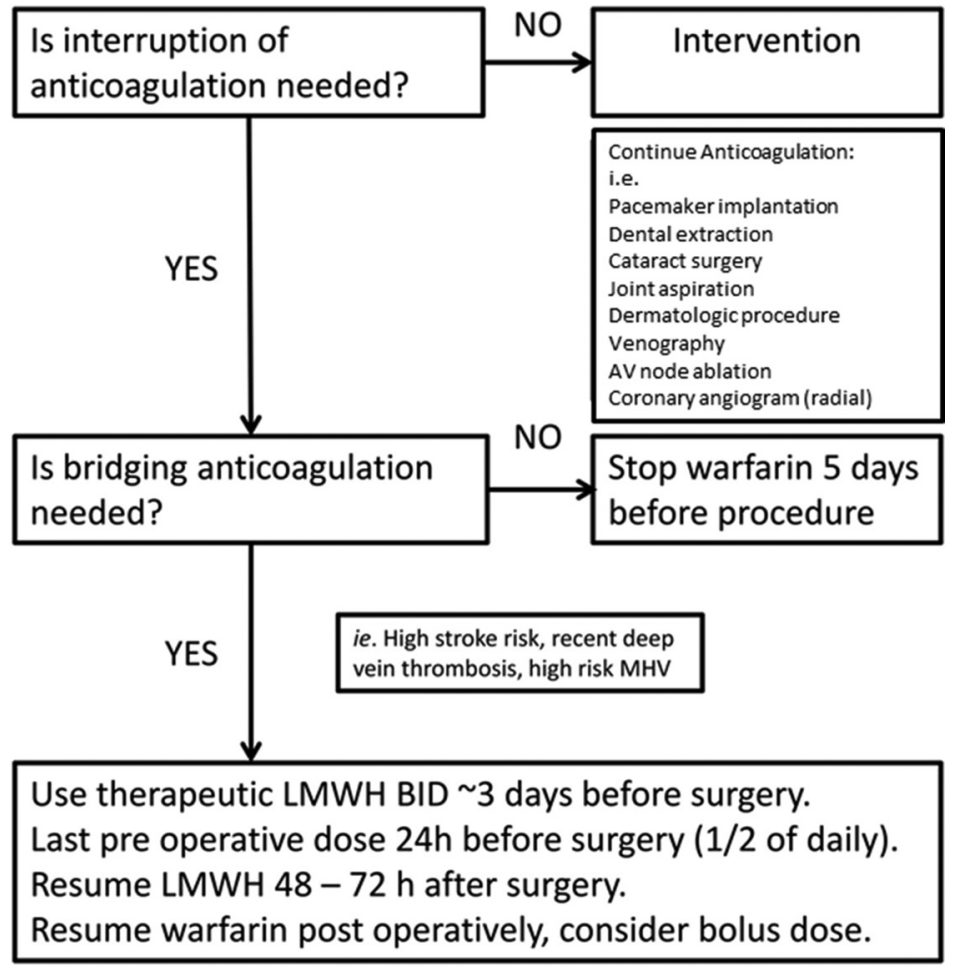

Figure 4 Suggested perioperative bridging protocol for patients on warfarin. Final action plan shall also consider bleeding risk including renal function. Venous thromboembolism prevention as needed in all patients. AV, atrioventricular; LMWH, low molecular weight heparin; MHV, mechanical heart valve. observational study of over 2000 patients with ACS in Sri Lanka found that less than $4 \%$ underwent primary PCI and only 63\% received fibrinolytic therapy, with a time to PCI $>90 \mathrm{~min}$ in $38 \%$ and a time to thrombolysis $>30 \mathrm{~min}$ in $58 \%$ of those receiving those therapies. ${ }^{3}$ Medical therapy with aspirin, clopidogrel and statins was provided in over $90 \%$ but beta-blockers were given to only $51 \%$ at hospital discharge. Patients treated at teaching hospitals underwent more procedures than those at non-teaching hospitals (figure 2).

In an editorial, Lodi-Junqueira and Ribeiro ${ }^{4}$ remind us that 'Cardiovascular disease (CVD) is now the leading cause of mortality and morbidity worldwide, accounting for approximately one-third of all deaths globally. While popular belief presumes that non-communicable diseases (NCDs) afflict mostly high-income populations, the evidence tells a very different story. Nearly $80 \%$ of NCD deaths occur in low-income and middle-income countries (LMICs). Ischaemic heart disease (IHD) is the leading component of the global CVD burden.' However, 'LMICs face the challenges of limited healthcare budgets and infrastructure as well as constrained professional health workforce capacity.' Even so, 'it is mandatory that each country, due to its population and resource diversities, appraises and monitors its ACS scenario to find its proper way to achieve the best clinical outcomes, adapting high income country (HIC) standards of care to the country reality.' Of course, in my view, HICs also need to ensure that known effective therapies for ACS are provided to all patients before considering the potential marginal benefits of advanced procedures such as CTO-PCI in a small subset of ACS patients.

Cardiac rehabilitation (CR) is associated with reduced cardiovascular mortality, lower rates of hospital readmission and improved physical quality of life in patients with coronary heart disease and heart failure. However, it remains uncertain whether CR actually improves physical activity (PA) levels in these patients. In a systematic review and meta-analysis of 40 randomised controlled trials, including almost 6000 cardiac patients, an improvement in PA was seen in $26 \%$ of studies with CR compared with controls. ${ }^{5}$ Metrics of PA showed improvement in steps/day and in the proportion of patients categorised as physically active (relative risk $1.55,95 \%$ CI 1.19 to $2.02, p=0.001)$. Unfortunately, there was a high risk of bias in these studies and quality of PA measurement was relatively poor suggesting that high quality trials are needed to determine 


\section{Discontinuation of DOAC Flow Diagram}

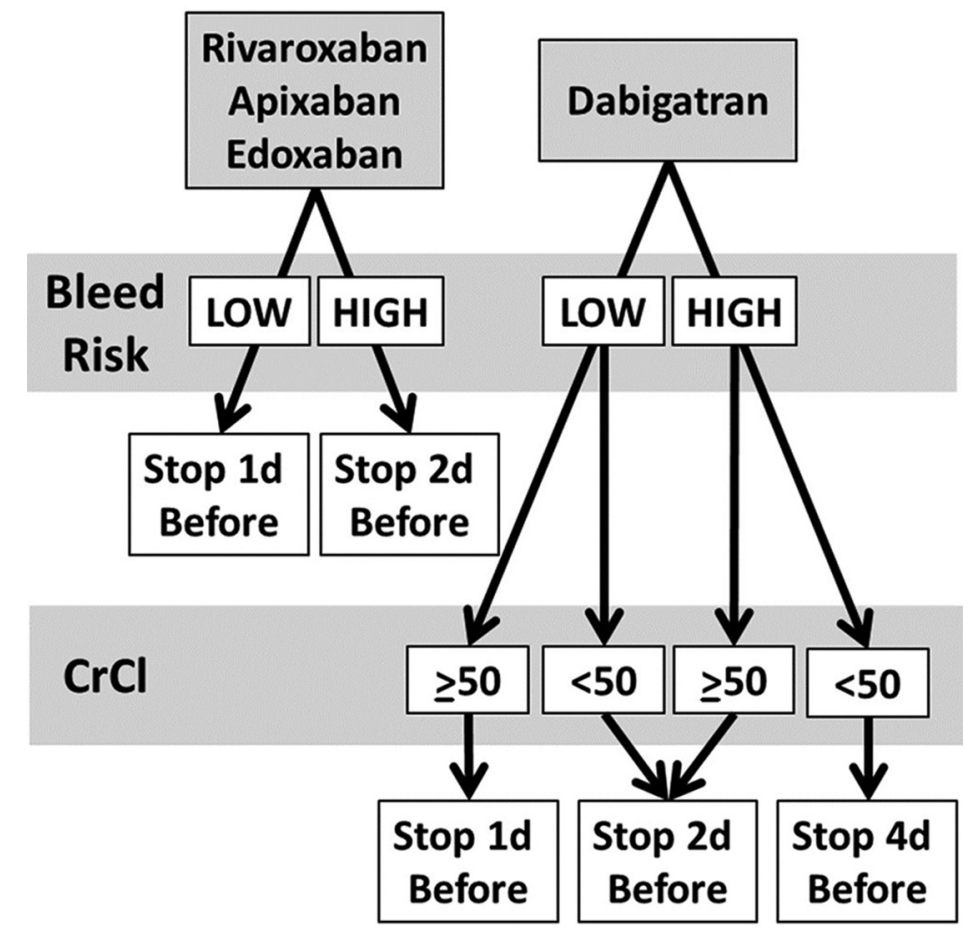

Figure 5 Suggested DOAC interruption schema based on pharmacodynamic approach. $\mathrm{CrCl}$, creatinine clearance; DOAC, direct oral anticoagulant.

if CR leads to significant improvement in PA in cardiac patients.

In a separate systematic review on the cost-effectiveness of $\mathrm{CR}$, most included studies found CR to be cost effective, based on reduced subsequent cardiac events and hospitalisations, with incremental cost-effectiveness ratios ranged from $\$ 1065$ to $\$ 71755$ per quality-adjusted life-year. ${ }^{6}$ The evidence for each component of CR was quite variable, particularly for psychological intervention and telehealth. ${ }^{7}$ While exercise was cost-effective across all studies, there was considerable uncertainly in the data (as found for measures of PA in the meta-analysis by Dibben and colleagues. ${ }^{5}$ This data will be useful for health policy decisions but additional studies are needed to determine the most cost-effective CR design.

The Education in Heart article ${ }^{8}$ in this issue provides an overview of management of anticoagulant and antiplatelet therapy in adults with heart disease who might require interruption of therapy for surgery or other procedures. This article addresses when interruption of therapy is needed which patients require perioperative 'bridging' with heparin (figure 4) and the optimal time frame and protocol for interruption of anticoagulation with a direct oral anticoagulant (figure 5).
Take a look at the Image Challenge in this issue. ${ }^{9}$ An unusual but interesting finding; I always learn something new in thinking about these multiple choice questions based on clinical images.

\section{Competing interests None declared.}

Patient consent Not required.

Provenance and peer review Commissioned; internally peer reviewed.

(c) Author(s) (or their employer(s)) 2018. No commercial re-use. See rights and permissions. Published by BMJ.

Check for updates

To cite Otto CM. Heart 2018;104:1385-1387.

Heart 2018;104:1385-1387

doi:10.1136/heartjnl-2018-313921

\section{REFERENCES}

1 Elias J, van Dongen IM, Råmunddal T, et al. EXPLORE investigators. Long-term impact of chronic total occlusion recanalisation in patients with ST-elevation myocardial infarction. Heart 2018;104:1432-8.

2 Stojkovic S, Milasinovic D. Chronic total occlusion percutaneous coronary intervention in clinical practice: novel grounds to be EXPLOREd. Heart 2018;104:1392-3.

3 Galappatthy P, Bataduwaarachchi VR, Ranasinghe P, et al. Management, characteristics and outcomes of patients with acute coronary syndrome in Sri Lanka. Heart 2018;104:1424-31.

4 Lodi-Junqueira L, Ribeiro AL. Tackling acute coronary syndrome in low-income and middle-income countries. Heart 2018;104:1390-1.

5 Dibben GO, Dalal HM, Taylor RS, et al. Cardiac rehabilitation and physical activity: systematic review and meta-analysis. Heart 2018:104:1394-402.

6 Shields GE, Wells A, Doherty P, et al. Costeffectiveness of cardiac rehabilitation: a systematic review. Heart 2018;104:1403-10.

7 Rawstorn JC, Gant N, Direito A, et al. Telehealth exercise-based cardiac rehabilitation: a systematic review and meta-analysis. Heart 2018;104:1403-10.

8 Tafur A, Douketis J. Perioperative management of anticoagulant and antiplatelet therapy. Heart 2018;104:1461-7.

9 Bradley AJ, Lewis JF, Choi AD, et al. Evaluation of an incidental cardiac finding in a patient with bronchitis. Heart 2018:104:1469-70. 